\title{
Development of Autonomous Intelligent Vehicle (AIV) Based on Voting Technique
}

\author{
M. R. Ghazali, M. S. Isa, W. I. Ibrahim, and S. H. Mohamed Noor
}

\begin{abstract}
This project is to develop an autonomous intelligent vehicle using voting technique. The technique is depending on goal-seeking behavior and obstacles avoidance behavior. An intelligent robot usually achieves the goal point safely without hitting any obstacles. The robot needs to consider about safety and goal directedness. Therefore, the voting technique becomes one of the approaches to overcome this problem. The voting technique takes an approach where multiple modules concurrently share control of the robot. It uses a scheme where each behavior votes for a discrete set of motor command, with vote zero is the least desired and vote one is the most desired action. This will solve the action of selection problem.
\end{abstract}

Index Terms-Voting technique, intelligent vehicle, goal-seeking behavior, obstacles avoidance behavior, action selection.

\section{INTRODUCTION}

TheMobile robot likely human behavior already develops nowdays. But, the capabilities of the robot are limited into certain behavior only. There have many inventor that create a different creation to increase the intelligent of mobile robot. Intelligence Vehicle robot is an automatic device which is capable of performing many complex tasks with seemingly human intelligence. In addition to setup mobile robot that have likely human behavior and look intelligent are challange task. Sometimes the robot cannot do all task that human can do. It is very difficult to explore and create mobile robot which is totally can do task like human. To overcome this, unmanned intelligent vehicle has been created.With unmanned task, this intelligent vehicle can explore and do some behavior that human can do. This intelligent vehicle generally have movement or behaviors. The control strategy is distributed among a set of specialized behaviors. The general behavior for human movement can be divided into two major behavior which depend on goal-seeking behavior and obstacle avoidance behavior [1]. Recently, intelligent vehicle have been used in limited number of task. With further research and development, intelligent vehicle can

Manuscript received December 20, 2013; revised March 26, 2014.

M. R. Ghazali is with the Robotics and Unmanned System Research Group (RUS), Faculty of Electrical and Electronics Engineering Universiti Malaysia Pahang, 26600 Pekan Pahang Malaysia (e-mail: riduwan@ ump.edu.my).

M. S. Isa was with Faculty of Electrical and Electronics Engineering Universiti Malaysia Pahang, 26600 Pekan Pahang.

W. I. Ibrahim is with the Sustainable Power Energy Research Group (SUPER), Faculty of Electrical and Electronics Engineering Universiti Malaysia Pahang, 26600 Pekan Pahang Malaysia (e-mail: wismail@ump.edu.my).

S. H. Mohamed Noor is with the Faculty of Manufacturing Engineering, Universiti Malaysia Pahang, 26600 Pekan, Pahang (e-mail: shaifulhakim@ump.edu.my). reduces the limitation and increase the capability to do more tasks. Today, most of intelligent vehicle or mobile robot has been seen in robotic field. In this paper, the development of autonomous intelligent vehicle based on voting technique has been discussed.

\section{PROJeCT BACKGROUND}

\section{A. Voting Technique}

The voting technique takes an approach where multiple modules concurrently share control of the robot. It uses a scheme where each behavior votes for a discrete set of motor commands and each set of motor command consist of the robot turn radius together with its speed. Various behaviors are designed for the mobile robot. These behaviors can be ranged from low-level behavior such as obstacle avoidance behavior, goal-seeking behavior to high-level behavior such as landmark detection behavior, path-planning behavior, and learning behavior. Each behavior is responsible for achieving some particular tasks. Each of them runs completely independently and asynchronously while generates a vote between 0 and 1 for every possible motor command.Vote zero is the least desired action and vote one is the most desired action. Each behavior is assigned a weight reflecting its priority in controlling the vehicle. An arbiter then performs command fusion to select the most appropriate action. Coordinate the commands from multiple behaviors that are active simultaneously [2].
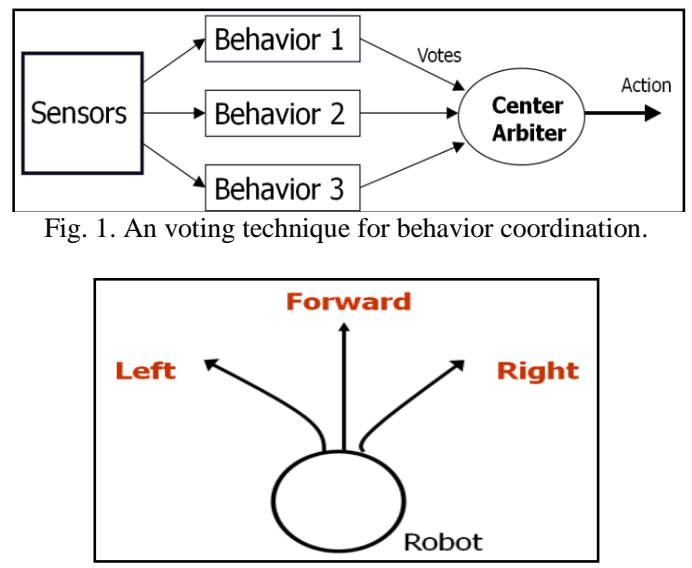

Fig. 2. Direction which can take after votes.

As can be seen in the Fig. 1, the behavior represent as a voter which is the person who sends their votes. The possible command set known as candidates which are the person who receives votes. In order to approval voting, voters can vote for as many candidates that they wish. Each behavior will vote for every candidate in the possible command set. Votes can combine together by usingweighted voting [3]. The 
preferences of some voters carry more weight than the preferences of other voters. Behavior with higher priority will carrying more weight. Here, command with the maximum vote will be chosen. In the Fig. 2 the direction can be made depending on the votes given.

\section{B. Ultrasonic Sensor (MaxSonar-EZ1)}

The MaxSonar-EZ1 offers very short to long-range detection and ranging, in an incredibly small package with ultra and low power consumption. The MaxSonar-EZ1 detects objects from 0 -inches to 254-inches (6.45-meters) and provides sonar range information from 6 -inches out to 254-inches with 1-inch resolution. Objects from 0 -inches to 6 -inches range as 6-inches. The interface output formats included are pulse width output, analog voltage output, and serial digital output [4].

\section{Compass Module Sensor (Honeywell HMC6352)}

The 'The Honeywell HMC6352 which is a fully integrated compass module that combines 2-axis magneto-resistive sensors with the required analog and digital support circuits, and algorithms for heading computation. By combining the sensor elements, processing electronics, and firmware in to a $6.5 \mathrm{~mm}$ by $6.5 \mathrm{~mm}$ by $1.5 \mathrm{~mm}$ LCC package, Honeywell offers a complete, ready to use electronic compass. This provides design engineers with the simplest solution to integrate high volume, cost effective compasses into wireless phones, consumer electronics, vehicle compassing, and antenna positioning. Honeywell continues to maintain product excellence and performance by introducing innovative solid-state magnetic sensor solutions. These are highly reliable, top performance products that are delivered when promised. Honeywell's magnetic sensor products provide real solutions you can count on.

The HMC6352 Integrated Compass Sensor circuit is composed of two magneto-resistive (MR) sensors with orthogonal orientation for sensing the horizontal components of the earth's magnetic field (0 to 630 milli-gauss), plus two amplifiers, a set/reset drive circuit, and a microprocessor $(\mu \mathrm{P})$. Best accuracy is obtained in clean magnetic environments (free air) and held level, or perpendicular to the gravitational direction. At worst case, each degree of tilt from a level orientation could add two degrees of compass heading error. Magnetic errors can be introduced if operated near strong magnetic sources such as microphone or speaker magnets, transformers in test equipment, and CRT deflection yokes in video displays/monitors. These magnetic errors can typically be reduced or eliminated by performing the calibration routine [5].

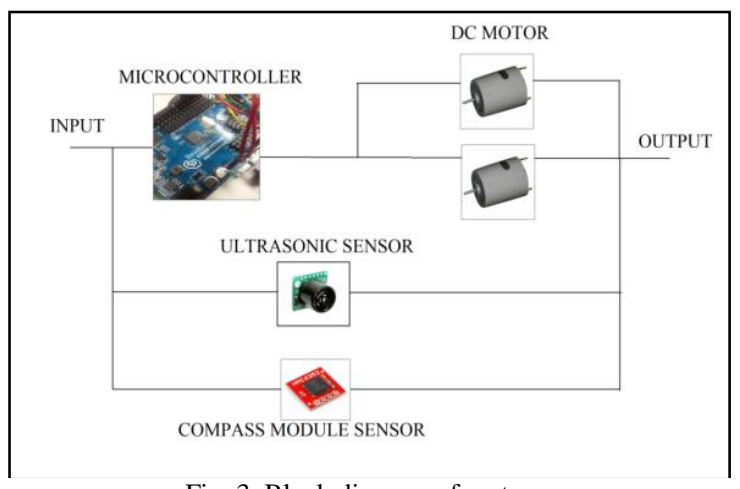

Fig. 3. Block diagram of system.

\section{MATH}

There are 3 steps for this project. The steps are design the hardware, software and testing the robot (method controlling).

Fig. 3 shown the close loop system consists of 3 set ultrasonic sensors and 1 compass module. The compass module was used as a system feedback. This project considers two behaviors which is goal seeking and obstacle avoiding behavior. The control method is dividing by 3 tasks. Task 1 is focusing on obstacle avoidance behavior while task 2 is focusing on goal seeking behavior. Task 3 focusing on combination of both behaviors with voting technique has been applied to make system more intelligent.

\section{A. Task 1}

Task 1 is about controlling the DC motor with three ultrasonic sensors. The purpose of this task is to move the IV robot in any direction, besides avoiding the obstacle that will be detected by ultrasonic sensor. Behaviour design for this task is for obstacle avoidance.

\section{B. Task 2}

The second task of this project is to control the DC motor with compass module sensor. The main objective of this task is to find the north $(\mathrm{N})$ direction. Then, IV robot need to move toward the direction.Behaviour design for this task is for goal seeking.

\section{Task 3}

Task 3 is to control the DC motor, ultrasonic sensor and compass module sensor based on voting technique. In this task, the IV robot needs to find the north direction. After found the north direction, IV robot needs to move forward. The IV robot needs to avoid any obstacle that will be detected by the ultrasonic sensor. The process continues and repeats the same step. Behaviours design for this task is a voting technique which is combination of goal seeking and obstacle avoidance.

\section{USING THE TEMPLATE}

\section{A. Task 1}

The purpose of this task is to move the IV robot in any direction and avoid the obstacle that will be detected on ultrasonic sensor. The IV robot move directly and can avoid any obstacle. If the IV robot did not found any obstacle, the robot moves forward. In generating a vote between 0 and 1 for every possible motor command, vote zero is the least desired action and vote one is the most desired action. The IV behavior can be determined by weight reflecting of ultrasonic sensor.But, the ultrasonic sensors need to put slightly higher to detect the obstacles clearly. Table I showed the result of task 1 based on sensor weighting division. The action is chosen by comparing each value of the sensors.

TABLE I: RESULT FOR TASK 1

\begin{tabular}{|c|c|c|c|}
\hline $\begin{array}{c}\text { Ultrasonic } \\
\text { sensor left } \\
\text { (S1) }\end{array}$ & $\begin{array}{c}\text { Ultrasonic } \\
\text { sensor } \\
\text { center(S2) }\end{array}$ & $\begin{array}{l}\text { Ultrasonic } \\
\text { sensor } \\
\text { right(S3) }\end{array}$ & Action \\
\hline 0.75 & 0.5 & 0.25 & Turn right \\
\hline 0.25 & 0.75 & 0.25 & Turn right \\
\hline 0.25 & 0.5 & 0.75 & Turn left \\
\hline 0 & 0 & 0 & Forward \\
\hline
\end{tabular}


Weight division for ultrasonic sensors:

$1=0 \mathrm{~cm}$ until $25 \mathrm{~cm}$

$0.75=26 \mathrm{~cm}$ until $40 \mathrm{~cm}$

$0.5=41 \mathrm{~cm}$ until $60 \mathrm{~cm}$

$0.25=61 \mathrm{~cm}$ until $70 \mathrm{~cm}$

$0=$ greater than $71 \mathrm{~cm}$

Fig. 4 shows that $\mathrm{S} 1$ able to detect the obstacle and send the vote 1 while $S 2$ and $S 3$ send the vote 0 . So, the IV will moving depended on programming (turn right). If the S1, S2 and S3 have same weight, so the movement of IV is depending on programming. This is because the task considers only 1 behavior. Fig. 5 and Fig. 6 show the different readings due to the position of the obstacles by the AIV.

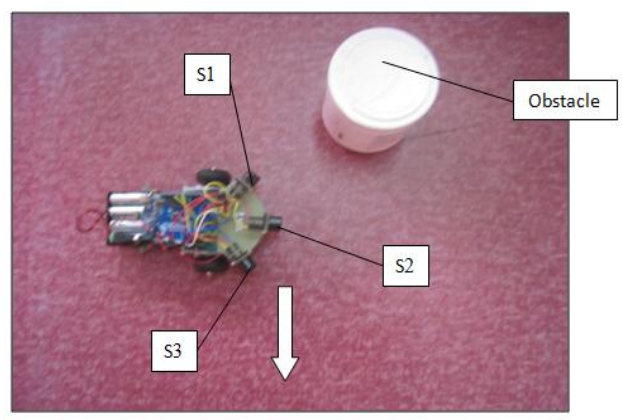

Fig. 4. S1 detect obstacle.

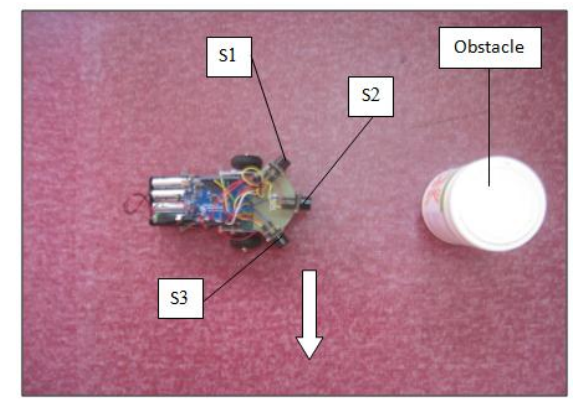

Fig. 5. S2 detect obstacle.

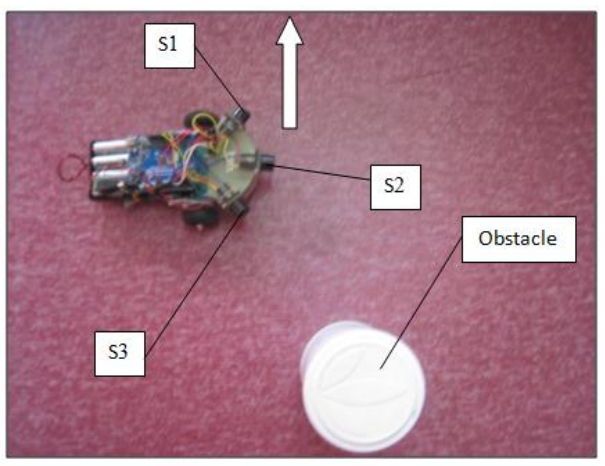

Fig. 6. S3 detect obstacle.

TABLE II: RESULT FOR TASK 2

\begin{tabular}{|l|l|}
\hline $\begin{array}{c}\text { Compass module } \\
\text { sensor }\left({ }^{\circ}\right)\end{array}$ & Action \\
\hline $0^{\circ}$ & Forward \\
\hline $90^{\circ}$ & Turn left \\
\hline $180^{\circ}$ & Turn right \\
\hline $270^{\circ}$ & Turn right \\
\hline
\end{tabular}

\section{B. Task 2}

Task 2 is about controlling the DC motor with compass module sensor. The main objective of this task is to find the north direction. Then, AIV robot move toward the direction. North direction is declared as $0^{\circ}$, regarding this task, IV robot can find north direction successfully. Fig. 7-Fig. 10 show the movement of IV based on different angle before achieved the $0^{\circ}$ (North direction). The result for the task 2 is shown in the Table II.

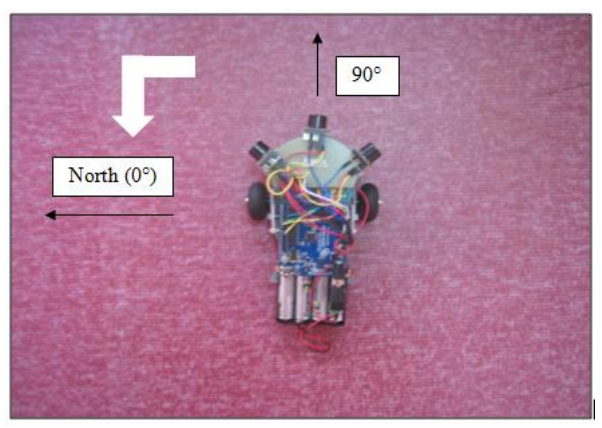

Fig. 7. IV movement when reading $90^{\circ}$.

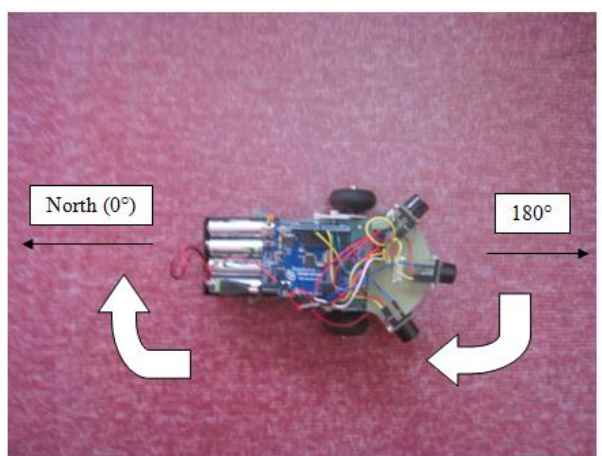

Fig. 8. IV movement when reading $180^{\circ}$.

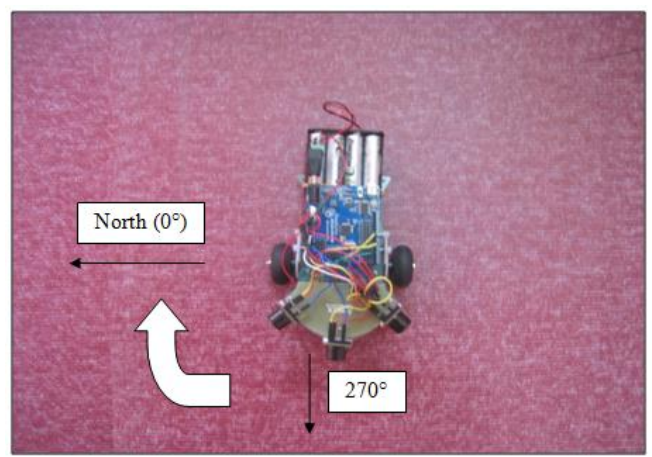

Fig. 9. IV movement when reading $270^{\circ}$.

\section{Task 3}

Task 3 is controlling the DC motor with feedback from three ultrasonic sensors and a compass module sensor by using voting technique to consider the goal seeking and obstacle avoidance behavior. The decision of movement is based on weight from sensors. Voting technique has been applied to IV robot to add an intelligent in decision making. In addition, IV robot was able to coordinate multiple behaviors and decision making. In this task, the IV robot need to find the north direction. Then, IV needs to move forward. While moving forward, the IV robot need to avoid any obstacle that detected by the ultrasonic sensors. The process continues with IV robot find the north direction again. If the weight has same, priority will be given to ultrasonic sensor. This is because obstacle avoidance behavior more important compare to goal seeking behavior. Table III showed the weight division comparison between ultrasonic sensors and 
compass module sensor and their action.

TABLE III: RESULT FOR TASK 3

\begin{tabular}{|c|c|c|c|c|}
\hline $\begin{array}{c}\text { Ultrasonic } \\
\text { sensor left } \\
(\mathrm{S} 1)\end{array}$ & $\begin{array}{c}\text { Ultraso } \\
\text { nic } \\
\text { sensor } \\
\text { center } \\
(\mathrm{S} 2)\end{array}$ & $\begin{array}{c}\text { Ultrasoni } \\
\text { c sensor } \\
\text { right (S3) }\end{array}$ & $\begin{array}{c}\text { Compass } \\
\text { module } \\
\text { sensor }\end{array}$ & Action \\
\hline 0.75 & 0.5 & 0.25 & 0.75 & $\begin{array}{c}\text { Turn } \\
\text { right }\end{array}$ \\
\hline 0.25 & 0.75 & 0.25 & 0.5 & $\begin{array}{c}\text { Turn } \\
\text { right }\end{array}$ \\
\hline 0.5 & 0.25 & 0.75 & 0.75 & \begin{tabular}{c} 
Turn left \\
\hline 0.25
\end{tabular} \\
\hline 0.25 & 0.25 & 0.25 & 0.5 & Turn left \\
\hline 0.25 & 0.5 & 0.25 & 0.75 & $\begin{array}{c}\text { Turn } \\
\text { right }\end{array}$ \\
\hline 0.5 & 0.5 & 0.25 & 1 & Forward \\
\hline & & 0.75 & 0.5 & Turn left \\
\hline & & & & 1 \\
\hline
\end{tabular}

Weight division for ultrasonic sensors:

$1=0 \mathrm{~cm}$ until $25 \mathrm{~cm}$

$0.75=26 \mathrm{~cm}$ until $40 \mathrm{~cm}$

$0.5=41 \mathrm{~cm}$ until $60 \mathrm{~cm}$

$0.25=61 \mathrm{~cm}$ until $70 \mathrm{~cm}$

$0=$ greater than $71 \mathrm{~cm}$

Weight division for compass module sensor:

$1=0^{\circ}$ until $10^{\circ}$

$0.75=11^{\circ}$ until $90^{\circ}$

$0.5=91^{\circ}$ until $179^{\circ}$

$0.25=180^{\circ}$ until $270^{\circ}$

$0=271^{\circ}$ until $359^{\circ}$

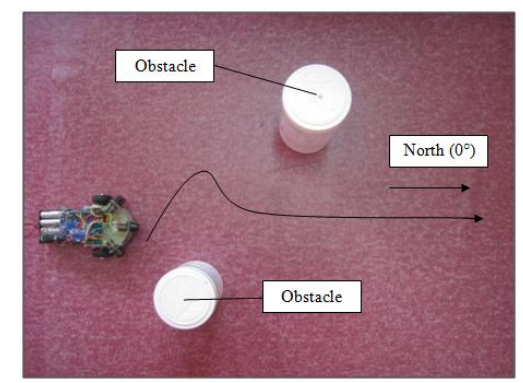

Fig. 10. IV robot finds north direction.

\section{CONCLUSION}

The AIV robot used 2 motor as a wheel to operate and find the setting direction.This prototype of AIV with Voting Technique was successfully designed and tested. In this project, the Ite aduino 2.2 was used as a main brain. The movement of AIV is based on goal point that has been setting in programming. The AIV has been tested based on task given.

\section{REFERENCES}

[1] B. B. K. Reddy, B. Kimiaghalam, A. H. A. C. Esterline, B. Sayyarrodsari, and N. S. Dugan, "Goal seeking with obstacle avoidance behavior for mobile robots," Electrical and Computer Engineering Department North Carolina, Agricultural and Technical State University, USA.

[2] T. C. Kwong, S. H. M. Amin, R. Mamat, and J. K. Tar, "Using voting technique in mobile robot behavior coordination for goal-directed navigation," Universiti Teknologi Malaysia, 2002.

[3] S. H. M. Amin, R. Mamat, and T. C. Kwong, "Behaviour based mobile robot navigation with dynamic weighted voting technique," in Cutting Edge Robotics, V. Kordic, A. Lazinica, and M. Merdan, Eds. ch. 13, 2007.

[4] Q. Y. Bao, S. M. Li, W. Y. Shang, and M. J. An, "A fuzzy behavior-based architecture for mobile robot navigation in unknown environments," Nanjing University of Aeronautics and Astronautics, Nanjing, P.R. China, 2009

[5] B. B. K. Reddy, "Goal seeking and obstacle avoidance algorithm for mobile robots," North Carolina A\&T State University, Greensboro, NC, 2003.

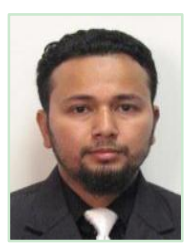

S. H. Mohamed Noor was born in Kota Bharu, in the state of Kelantan, east-coast of Malaysia. He graduated from Universite Paris Ouest La Defence, Paris, France in 2010 in master research (energy and aerospace propulsion engineering). He obtained his bachelor degree (liscence) from Universite Paris 12, France in 2007 in maintenance engineering (aeronautical), and obtained his Diploma in Thermal and Energy Engineering from Universite Blaise Pascal 2, Clermont Ferrand, France in 2005. His main specialties are the aerospace, aeronautical and heat engineering studies.

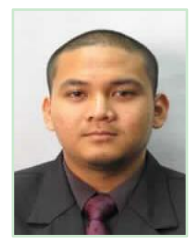

M. R. Ghazali was born in Pahang, east-coast of Malaysia and obtained his master's degree at Universiti Teknologi Malaysia in 2010 and his bachelor's degree in 2007 in the same university. M.R Ghazali is with the Robotics and Unmanned System Research Group (RUS), of the Faculty of Electrical and Electronics,Universiti Malaysia Pahang.

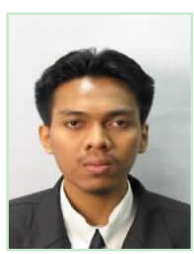

W. I. Ibrahim was born in Kelantan, east-coast of Malaysia and obtained his master's degree at Universiti Teknologi Malaysia in 2010 in the power generated system, electrical engineering. He graduated in Universiti Malaysia Pahang in bachelor's degree of electrical and elctronic engineering in 2007. W.I. Ibrahim is with the Sustainable Power Energy Research Group (SUPER) Faculty of Electrical and Electronics Engineering Universiti Malaysia Pahang. 